\title{
Subsystem Identification of Multivariable Feedback and Feedforward Systems
}

\author{
Xingye Zhang and Jesse B. Hoagg \\ Department of Mechanical Engineering, University of Kentucky, Lexington, KY 40506-0503
}

\begin{abstract}
We present a frequency-domain technique for identifying multivariable feedback and feedforward subsystems that are interconnected with a known subsystem. This subsystem identification algorithm uses closed-loop input-output data, but no other system signals are assumed to be measured. In particular, neither the feedback signal nor the outputs of the unknown subsystems are assumed to be measured. We use a candidate-pool approach to identify the feedback and feedforward transfer function matrices, while guaranteeing asymptotic stability of the identified closed-loop transfer function matrix. The main analytic result shows that if the data noise is sufficiently small and the candidate pool is sufficiently dense, then the parameters of the identified feedback and feedforward transfer function matrices are arbitrarily close to the true parameters.
\end{abstract}

\section{Introduction}

Subsystem identification (SSID) is the process of building empirical models of unknown dynamic subsystems, which are interconnected with known dynamic subsystems. These connections can be series, parallel, or feedback. SSID relies on measured data to identify the unknown subsystems. However, not all input and output signals to the unknown subsystems are necessarily accessible, that is, available for measurement.

This paper is concerned with closed-loop SSID of unknown feedback and feedforward subsystems interconnected with a known subsystem as shown in Figure 1. The exogenous input $r$ and closed-loop output $y$ are measured, whereas internal signals $u$ and $v$ are not assumed to be accessible. We note that closed-loop SSID is distinct from the well-studied problem of system identification in closed loop [1-4]. Specifically, in SSID, the unknown subsystems have inputs or outputs that are inaccessible.

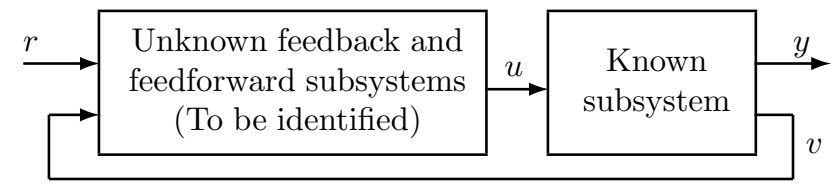

Fig. 1. The unknown feedback and feedforward subsystems are to be identified using the measured data $r$ and $y$. The internal signals $u$ and $v$ are inaccessible.

SSID has applications in biology and physics as well as human-in-the-loop systems. For example, many biological systems are modeled by the interconnection of sub-

Email addresses: xingyezhang86@gmail.com (Xingye Zhang), jhoagg@engr.uky.edu (Jesse B. Hoagg),

This work was supported by the National Science Foundation through CMMI-1405257. systems, which may be unknown and have inaccessible inputs and outputs [5]. Similarly, physical systems are often modeled by a composition of subsystems, which are based on either physical laws or empirical information. For example, in [6], a large-scale physics-based model of the global ionosphere-thermosphere is improved by using measured data to estimate thermal conductivity, which can be regarded as an unknown feedback subsystem. In this application, the output of the unknown subsystem is inaccessible.

SSID also has application to modeling human behavior. For example, there is interest in modeling human-inthe-loop behavior for applications such as aircraft [7-10] and automobiles [11-13]. In addition, SSID methods can be used to model human behavior in motor control experiments, which study human learning [14-17].

Closed-loop SSID of feedback and feedforward models is considered in $[6,18-20]$. However, the identified feedback and feedforward models obtained from the methods in $[6,18-20]$ can result in unstable closed-loop dynamics. To address closed-loop stability, [17] presents an SSID technique that guarantees asymptotic stability of the identified closed-loop transfer function. The approach in [17] applies to single-input single-output (SISO) subsystems and requires that the measured closed-loop output $y$ is the same as the feedback $v$.

The new contribution of this paper is a closed-loop SSID method that: i) identifies multi-input multi-output (MIMO) feedback and feedforward subsystems; ii) allows for a measured output $y$ that is not necessarily the same as the feedback $v$; and iii) guarantees asymptotic stability of the identified closed-loop transfer function matrix. This paper adopts techniques from [17] but goes beyond the previous work by addressing MIMO subsystems and allowing for the measured output $y$ to differ from the feedback $v$. Furthermore, the discrete- 
time SSID approach in this paper can improve computational efficiency relative to the continuous-time approaches in [17]. In this paper, the feedforward subsystem model is parameterized as a finite impulse response (FIR) transfer function matrix, which can improve computational efficiency as discussed in Section 7. To accomplish i)-iii), we use a candidate-pool approach. Our main analytic result shows that if the data noise is sufficiently small and the candidate pool is sufficiently dense, then the parameters of the identified feedback and feedforward transfer function matrices are arbitrarily close to the true parameters.

\section{Notation}

Let $\mathbb{F}$ be either $\mathbb{R}$ or $\mathbb{C}$. Then, $x_{(i)}$ denotes the $i$ th component of $x \in \mathbb{F}^{n}$, and $A_{(i, j)}$ denotes the $(i, j)$ entry of $A \in \mathbb{F}^{m \times n}$. Let $\|\cdot\|$ be a norm on $\mathbb{F}^{m \times n}$, and let $\|\cdot\|_{2}$ be the two-norm on $\mathbb{F}^{n}$. Next, let $A^{*}$ denote the complex conjugate transpose of $A \in \mathbb{F}^{m \times n}$, and define $\|A\|_{\mathrm{F}} \triangleq$ $\sqrt{\operatorname{tr} A^{*} A}$, which is the Frobenius norm of $A \in \mathbb{F}^{m \times n}$. Let $A^{\mathrm{A}}$ denote the adjugate of $A \in \mathbb{F}^{m \times n}$.

Let $\operatorname{vec} A$ be the vector in $\mathbb{F}^{m n}$ formed by stacking the columns of $A \in \mathbb{F}^{m \times n}$. Let vec $^{-1}$ be the inverse vec operator, that is, $\operatorname{vec}^{-1}(\operatorname{vec} A)=A$. Let $A \otimes B$ denote the Kronecker product of $A \in \mathbb{F}^{m \times n}$ and $B \in \mathbb{F}^{k \times l}$.

Let $\mathbb{R}[z]$ denote the set of polynomials with coefficients in $\mathbb{R}$, and let $\mathbb{R}^{m \times n}[z]$ denote the set of $m \times n$ polynomial matrices, that is, the set of matrix functions $P: \mathbb{C} \rightarrow$ $\mathbb{C}^{m \times n}$ whose entries are elements in $\mathbb{R}[z]$. The degree of the polynomial $p \in \mathbb{R}[z]$ is denoted by $\operatorname{deg} p$, and the degree of the polynomial matrix $P \in \mathbb{R}^{m \times n}[z]$ is denoted by $\operatorname{deg} P \triangleq \max _{i=1, \ldots, m ; j=1, \ldots, n} \operatorname{deg} P_{(i, j)}$.

Define the open ball of radius $\epsilon>0$ centered at $c \in$ $\mathbb{F}^{m \times n}$ by $\mathbb{B}_{\epsilon}(c) \triangleq\left\{x \in \mathbb{F}^{m \times n}:\|x-c\|<\epsilon\right\}$. Let $\mathbb{Z}^{+}$ denote the set of positive integers.

Definition 1 Let $\Delta \subseteq \mathbb{F}^{m \times n}$ be bounded and contain no isolated points. For all $j \in \mathbb{Z}^{+}$, let $\Delta_{j} \subseteq \Delta$ be a finite set. Then, $\left\{\Delta_{j}\right\}_{j=1}^{\infty}$ converges to $\Delta$ if for each $x \in \Delta$, there exists a sequence $\left\{x_{j}: x_{j} \in \Delta_{j}\right\}_{j=1}^{\infty}$ such that for all $\epsilon>0$, there exists $L \in \mathbb{Z}^{+}$such that for all $j>L$, $x_{j} \in \mathbb{B}_{\epsilon}(x)$.

\section{Problem Formulation}

Let $G_{y}: \mathbb{C} \rightarrow \mathbb{C}^{n \times m}$ and $G_{v}: \mathbb{C} \rightarrow \mathbb{C}^{l \times m}$ be real rational transfer function matrices, and consider the linear time-invariant system

$$
\begin{aligned}
& y(z)=G_{y}(z)\left[u(z)+\gamma_{u}(z)\right]+\gamma_{y}(z), \\
& v(z)=G_{v}(z)\left[u(z)+\gamma_{u}(z)\right]
\end{aligned}
$$

where $y(z) \in \mathbb{C}^{n}, \gamma_{y}(z) \in \mathbb{C}^{n}, u(z) \in \mathbb{C}^{m}, \gamma_{u}(z) \in \mathbb{C}^{m}$, and $v(z) \in \mathbb{C}^{l}$ are the $z$-transforms of the output, output noise, control, control noise, and feedback, respectively. The control $u$ is generated by feedback and feedforward as shown in Figure 2. Let $G_{\mathrm{ff}}, G_{\mathrm{fb}}: \mathbb{C} \rightarrow \mathbb{C}^{m \times l}$ be real rational transfer function matrices, and consider the control

$$
u(z)=G_{\mathrm{ff}}(z)\left[r(z)+\gamma_{r}(z)\right]+G_{\mathrm{fb}}(z)\left[e(z)+\gamma_{e}(z)\right],
$$

where $r(z) \in \mathbb{C}^{l}$ is the exogenous input, $\gamma_{r}(z) \in \mathbb{C}^{l}$ is the feedforward noise, $e(z) \triangleq r(z)-v(z)$ is the error, and $\gamma_{e}(z) \in \mathbb{C}^{l}$ is the error noise. We assume that $G_{\mathrm{ff}}$ is asymptotically stable, that is, the poles of $G_{\mathrm{ff}}$ are contained in the open unit disk. The closed-loop system obtained from (1)-(3) is

$$
y(z)=\tilde{G}(z) r(z)+\gamma(z),
$$

where

$$
\tilde{G} \triangleq G_{y}\left(I_{m}+G_{\mathrm{fb}} G_{v}\right)^{-1}\left(G_{\mathrm{fb}}+G_{\mathrm{ff}}\right)
$$

is assumed to be asymptotically stable, and the noise is

$$
\begin{aligned}
& \gamma \triangleq G_{y}\left(I_{m}+G_{\mathrm{fb}} G_{v}\right)^{-1}\left(G_{\mathrm{ff}} \gamma_{r}+G_{\mathrm{fb}} \gamma_{e}-G_{\mathrm{fb}} G_{v} \gamma_{u}\right) \\
& +G_{y} \gamma_{u}+\gamma_{y} .
\end{aligned}
$$

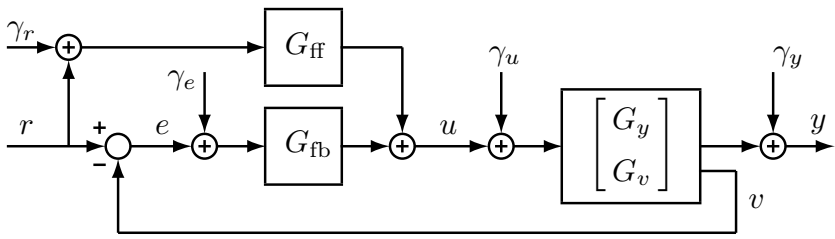

Fig. 2. The input $r$ and output $y$ are measured, but all internal signals and the noises are unmeasured.

Let $N \in \mathbb{Z}^{+}$be the number of frequency response data, and define $\mathcal{N} \triangleq\{1,2, \ldots, N\}$. For all $k \in \mathcal{N}$, let $\theta_{k} \in[0, \pi]$, where $\theta_{1}<\cdots<\theta_{N}$. Define the closed-loop frequency response data

$$
H\left(\theta_{k}\right) \triangleq \tilde{G}\left(e^{\jmath \theta_{k}}\right)+\Gamma\left(e^{\jmath \theta_{k}}\right) \in \mathbb{C}^{n \times l},
$$

where $\Gamma: \mathbb{C} \rightarrow \mathbb{C}^{n \times l}$ is such that, for all $i \in\{1,2, \ldots, n\}$ and all $j \in\{1,2, \ldots, l\}, \Gamma_{(i, j)} \triangleq \gamma_{(i)} / r_{(j)}$. Define the noise matrix

$$
\eta_{*} \triangleq\left[\Gamma\left(\sigma_{1}\right) \cdots \Gamma\left(\sigma_{N}\right)\right] \in \mathbb{C}^{n \times l N} .
$$

This paper presents an SSID method to identify $G_{\mathrm{ff}}$ and $G_{\mathrm{fb}}$ under the assumption that $G_{y}, G_{v}$, and $\left\{H\left(\theta_{k}\right)\right\}_{k=1}^{N}$ are known. For each $k \in \mathcal{N}, H\left(\theta_{k}\right)$ can be calculated from $y$ and $r$ as $H_{(i, j)}\left(\theta_{k}\right)=$ $y_{(i)}\left(e^{\jmath \theta_{k}}\right) / r_{(j)}\left(e^{\jmath \theta_{k}}\right)$. Thus, $\left\{H\left(\theta_{k}\right)\right\}_{k=1}^{N}$ can be obtained from the accessible signals $r$ and $y$, and does not depend on the internal signals (e.g., $u$ and $v$ ) or the noise signals $\gamma_{r}, \gamma_{e}, \gamma_{u}$, and $\gamma_{y}$, which are not assumed to be measured.

We assume that $G_{\mathrm{ff}}$ is FIR. Thus, we can express the feedforward transfer function matrix as $G_{\mathrm{ff}}(z)=$ $z^{-n_{\mathrm{ff}}} N_{\mathrm{ff}}(z)$, where $N_{\mathrm{ff}} \in \mathbb{R}^{m \times l}[z]$ and $n_{\mathrm{ff}} \triangleq \operatorname{deg} N_{\mathrm{ff}}$. Since $G_{\mathrm{ff}}$ is asymptotically stable, it follows that for sufficiently large order $n_{\mathrm{ff}}, G_{\mathrm{ff}}$ can approximate an infinite impulse response (IIR) transfer function matrix to arbitrary accuracy evaluated along the unit circle. Thus, the assumption that $G_{\mathrm{ff}}$ is FIR does not significantly restrict 
the class of feedforward behavior. The SSID approach in this paper can also be used with an IIR feedforward model, but using an FIR feedforward model improves computational efficiency as discussed in Section 7.

Let $G_{y}$ and $G_{v}$ have the right-matrix-fraction descriptions $G_{y}=N_{y} D^{-1}$ and $G_{v}=N_{v} D^{-1}$, and let $G_{\mathrm{fb}}$ have the left-matrix-fraction description $G_{\mathrm{fb}}=D_{\mathrm{fb}}^{-1} N_{\mathrm{fb}}$, where $N_{y} \in \mathbb{R}^{n \times m}[z], N_{v} \in \mathbb{R}^{l \times m}[z], N_{\mathrm{fb}} \in \mathbb{R}^{m \times l}[z]$, and $D, D_{\mathrm{fb}} \in \mathbb{R}^{m \times m}[z]$. Without loss of generality, we assume that $D$ and $D_{\mathrm{fb}}$ are monic. Thus, (4) can be expressed as

$$
\tilde{G}(z)=N_{y}(z) \tilde{D}^{-1}(z)\left[N_{\mathrm{fb}}(z)+z^{-n_{\mathrm{ff}}} D_{\mathrm{fb}}(z) N_{\mathrm{ff}}(z)\right],
$$

where

$$
\tilde{D} \triangleq D_{\mathrm{fb}} D+N_{\mathrm{fb}} N_{v} \in \mathbb{R}^{m \times m}[z] .
$$

Define $d \triangleq \operatorname{deg} D, d_{\mathrm{fb}} \triangleq \operatorname{deg} D_{\mathrm{fb}}, n_{y} \triangleq \operatorname{deg} N_{y}, n_{v} \triangleq$ $\operatorname{deg} N_{v}$, and $n_{\mathrm{fb}} \triangleq \operatorname{deg} N_{\mathrm{fb}}$. We make the following assumptions:

(A1) $d+d_{\mathrm{fb}}>n_{v}+n_{\mathrm{fb}}$.

(A2) $N>n_{y}+(m-1)\left(d+d_{\mathrm{fb}}\right)+d_{\mathrm{fb}}+n_{\mathrm{ff}}$.

(A3) If $\lambda \in \mathbb{C}$ and $\operatorname{det} \tilde{D}(\lambda)=0$, then $|\lambda|<1$.

Assumption (A1) states that $G_{\mathrm{fb}} G_{v}$ is strictly proper. Assumption (A2) requires that the number $N$ of frequency response data points is sufficiently large. Assumption (A3) implies that $\tilde{G}$ is asymptotically stable. We also assume that $n_{\mathrm{ff}}, d_{\mathrm{fb}}$, and $n_{\mathrm{fb}}$ are known.

To formulate the SSID problem, define $a \triangleq l\left(n_{\mathrm{fb}}+\right.$ $1)+m d_{\mathrm{fb}}$ and $b \triangleq m\left(n_{\mathrm{ff}}+1\right)$, and consider the functions $\mathcal{N}_{\mathrm{ff}}: \mathbb{C} \times \mathbb{R}^{b \times l} \rightarrow \mathbb{C}^{m \times l}, \mathcal{N}_{\mathrm{fb}}: \mathbb{C} \times \mathbb{R}^{a \times m} \rightarrow \mathbb{C}^{m \times l}$, $\mathcal{D}_{\mathrm{fb}}: \mathbb{C} \times \mathbb{R}^{a \times m} \rightarrow \mathbb{C}^{m \times m}$ given by

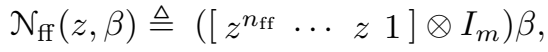

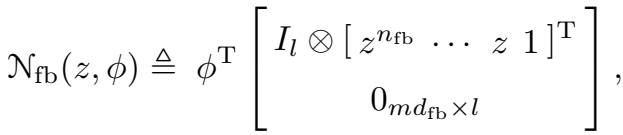

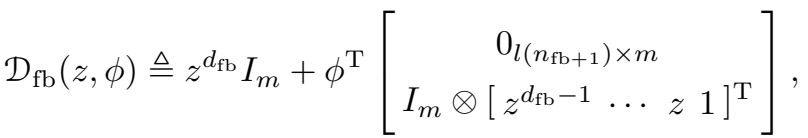

where $\beta \in \mathbb{R}^{b \times l}$ contains the unknown parameters of $\mathcal{N}_{\mathrm{ff}}$, and $\phi \in \mathbb{R}^{a \times m}$ contains the unknown parameters of $\mathcal{N}_{\mathrm{fb}}$ and $\mathcal{D}_{\mathrm{fb}}$. Consider $\mathcal{G}_{\mathrm{ff}}: \mathbb{C} \times \mathbb{R}^{b \times l} \rightarrow \mathbb{C}^{m \times l}$ and $\mathcal{G}_{\mathrm{fb}}: \mathbb{C} \times \mathbb{R}^{a \times m} \rightarrow \mathbb{C}^{m \times l}$ given by

$$
\begin{aligned}
& \mathcal{G}_{\mathrm{ff}}(z, \beta) \triangleq z^{-n_{\mathrm{ff}}} \mathcal{N}_{\mathrm{ff}}(z, \beta), \\
& \mathcal{G}_{\mathrm{fb}}(z, \phi) \triangleq \mathcal{D}_{\mathrm{fb}}^{-1}(z, \phi) \mathcal{N}_{\mathrm{fb}}(z, \phi),
\end{aligned}
$$

which, for each $\beta \in \mathbb{R}^{b \times l}$ and $\phi \in \mathbb{R}^{a \times m}$, are real rational transfer function matrices.

Let $\beta_{*} \in \mathbb{R}^{b \times l}$ and $\phi_{*} \in \mathbb{R}^{a \times m}$ be such that,

$$
\begin{aligned}
& N_{\mathrm{ff}}(z) \equiv \mathcal{N}_{\mathrm{ff}}\left(z, \beta_{*}\right), \\
& N_{\mathrm{fb}}(z) \equiv \mathcal{N}_{\mathrm{fb}}\left(z, \phi_{*}\right), \\
& D_{\mathrm{fb}}(z) \equiv \mathcal{D}_{\mathrm{fb}}\left(z, \phi_{*}\right) .
\end{aligned}
$$

Thus, $\mathcal{G}_{\mathrm{ff}}\left(z, \beta_{*}\right) \equiv G_{\mathrm{ff}}(z)$ and $\mathcal{G}_{\mathrm{fb}}\left(z, \phi_{*}\right) \equiv G_{\mathrm{fb}}(z)$. Consider $\tilde{\mathcal{G}}: \mathbb{C} \times \mathbb{R}^{b \times l} \times \mathbb{R}^{a \times m} \rightarrow \mathbb{C}^{n \times l}$ given by

$$
\begin{aligned}
\tilde{\mathcal{G}}(z, \beta, \phi) \triangleq & N_{y}(z) \tilde{\mathcal{D}}^{-1}(z, \phi)\left[\mathcal{N}_{\mathrm{fb}}(z, \phi)\right. \\
& \left.+z^{-n_{\mathrm{ff}}} \mathcal{D}_{\mathrm{fb}}(z, \phi) \mathcal{N}_{\mathrm{ff}}(z, \beta)\right],
\end{aligned}
$$

where

$$
\tilde{\mathcal{D}}(z, \phi) \triangleq \mathcal{D}_{\mathrm{fb}}(z, \phi) D(z)+\mathcal{N}_{\mathrm{fb}}(z, \phi) N_{v}(z) .
$$

Note that $\tilde{\mathcal{G}}(z, \beta, \phi)$ is the closed-loop transfer function obtained from $\beta$ and $\phi$. Thus, $\tilde{\mathcal{G}}\left(z, \beta_{*}, \phi_{*}\right) \equiv \tilde{G}(z)$.

Our objective is to determine $\beta$ and $\phi$ such that $\mathcal{G}_{\mathrm{ff}}$ and $\mathcal{G}_{\mathrm{fb}}$ approximate $G_{\mathrm{ff}}$ and $G_{\mathrm{fb}}$, respectively. To achieve this objective, we seek to minimize

$$
J(\beta, \phi)=\sum_{k=1}^{N}\left\|\tilde{\mathcal{G}}\left(e^{\jmath \theta_{k}}, \beta, \phi\right)-H\left(\theta_{k}\right)\right\|_{\mathrm{F}}^{2},
$$

subject to the constraint that $\tilde{\mathcal{D}}(z, \phi)$ is asymptotically stable, that is, $\phi \in \mathcal{S}$, where

$$
\begin{aligned}
& \mathcal{S} \triangleq\left\{\phi \in \mathbb{R}^{a \times m}:\right. \text { if } \lambda \in \mathbb{C} \text { and } \operatorname{det} \tilde{\mathcal{D}}(\lambda, \phi)=0, \\
&\text { then }|\lambda|<1\} .
\end{aligned}
$$

The cost (8) measures the difference between the data $\left\{H\left(\theta_{k}\right)\right\}_{k=1}^{N}$ and the closed-loop transfer function matrix obtained from the estimates $\mathcal{G}_{\mathrm{ff}}$ and $\mathcal{G}_{\mathrm{fb}}$. The cost (8) and constraint $\phi \in \mathcal{S}$ are nonlinear and nonconvex in $(\beta, \phi)$. If $\Gamma\left(e^{\jmath \theta_{k}}\right) \equiv 0$, then $J\left(\beta_{*}, \phi_{*}\right)=0$.

\section{Subsystem Identification Algorithm}

We now develop an SSID algorithm to estimate $G_{\text {ff }}$ and $G_{\mathrm{fb}}$. For each $k \in \mathcal{N}$, define $\sigma_{k} \triangleq e^{\jmath \theta_{k}}$, and define

$$
\begin{aligned}
& \mathcal{A}_{k}(\phi) \triangleq \sigma_{k}^{-n_{\mathrm{ff}}} N_{y}\left(\sigma_{k}\right) \tilde{\mathcal{D}}^{-1}\left(\sigma_{k}, \phi\right) \mathcal{D}_{\mathrm{fb}}\left(\sigma_{k}, \phi\right) \nu\left(\sigma_{k}\right), \\
& \mathcal{B}_{k}(\phi) \triangleq N_{y}\left(\sigma_{k}\right) \tilde{\mathcal{D}}^{-1}\left(\sigma_{k}, \phi\right) \mathcal{N}_{\mathrm{fb}}\left(\sigma_{k}, \phi\right)-H\left(\theta_{k}\right),
\end{aligned}
$$

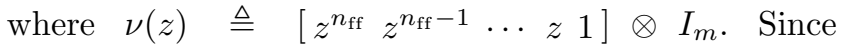
$\operatorname{vec}(A B)=(I \otimes A) \operatorname{vec} B$, it follows from $(6)-(10)$ that

$$
\begin{aligned}
J(\beta, \phi) & =\sum_{k=1}^{N}\left\|\mathcal{A}_{k}(\phi) \beta+\mathcal{B}_{k}(\phi)\right\|_{\mathrm{F}}^{2} \\
& =\sum_{k=1}^{N}\left\|\left[I_{l} \otimes \mathcal{A}_{k}(\phi)\right] \operatorname{vec} \beta+\operatorname{vec} \mathcal{B}_{k}(\phi)\right\|_{2}^{2} \\
& =[\operatorname{vec} \beta]^{\mathrm{T}} \Omega_{2}(\phi) \operatorname{vec} \beta+\Omega_{1}^{\mathrm{T}}(\phi) \operatorname{vec} \beta+\Omega_{0}(\phi),
\end{aligned}
$$

where

$$
\begin{aligned}
& \Omega_{0}(\phi) \triangleq \sum_{k=1}^{N}\left\|\mathcal{B}_{k}(\phi)\right\|_{\mathrm{F}}^{2} \in \mathbb{R} \\
& \Omega_{1}(\phi) \triangleq 2 \operatorname{Re} \sum_{k=1}^{N} \operatorname{vec}\left(\mathcal{A}_{k}^{*}(\phi) \mathcal{B}_{k}(\phi)\right) \in \mathbb{R}^{l b} \\
& \Omega_{2}(\phi) \triangleq I_{l} \otimes \operatorname{Re} \sum_{k=1}^{N} \mathcal{A}_{k}^{*}(\phi) \mathcal{A}_{k}(\phi) \in \mathbb{R}^{l b \times l b} .
\end{aligned}
$$


For the remaining of this paper, we assume that for all $\phi \in \mathcal{S}, \Omega_{2}(\phi)$ is positive definite. The following result provides a sufficient condition such that $\Omega_{2}(\phi)$ is positive definite. The proof is in Appendix A.

Proposition 1 Consider $\Omega_{2}$ given by (14), where (A1) and (A2) are satisfied. Assume that $\max _{z \in \mathbb{C}} \operatorname{rank} N_{y}(z)$ $=m \leq n$. Then, for all $\phi \in \mathcal{S}, \Omega_{2}(\phi)$ is positive definite.

Proposition 1 implies that if $G_{y}$ is SISO (i.e., $m=n=$ $1)$, then for all $\phi \in \mathcal{S}, \Omega_{2}(\phi)$ is positive definite. Proposition 1 also implies that if the number $m$ of measurements does not exceed the number $n$ of controls and $G_{y}$ has full normal rank, then for all $\phi \in \mathcal{S}, \Omega_{2}(\phi)$ is positive definite.

The next result shows that for each $\phi \in \mathcal{S}, J(\beta, \phi)$ has a unique global minimizer [21, Chap. 1].

Proposition 2 Consider $J$ given by (11), where (A1) and (A2) are satisfied. Let $\phi \in \mathcal{S}$ and define

$$
\beta_{\min } \triangleq-\frac{1}{2} \operatorname{vec}^{-1}\left[\Omega_{2}^{-1}(\phi) \Omega_{1}(\phi)\right] \in \mathbb{R}^{b \times l} .
$$

Let $\beta \in \mathbb{R}^{b \times l} \backslash\left\{\beta_{\min }\right\}$. Then, $J\left(\beta_{\min }, \phi\right)<J(\beta, \phi)$.

Let $\Phi \subseteq \mathcal{S}$ be a set with $M$ elements. We call $\Phi$ the candidate pool. We now create a candidate sequence using the $M$ elements in $\Phi$. For all $i, j \in \mathcal{M} \triangleq\{1,2, \ldots, M\}$, let $\phi_{i}, \phi_{j} \in \Phi$ be such that if $i \neq j$, then $\phi_{i} \neq \phi_{j}$. Now, for all $i \in \mathcal{M}$, define the quadratic cost function

$$
\mathcal{J}_{i}(\beta) \triangleq J\left(\beta, \phi_{i}\right) .
$$

Since $\phi_{1}, \ldots, \phi_{M} \in \Phi \subseteq \mathcal{S}$, it follows that $\Omega_{2}\left(\phi_{1}\right), \ldots$, $\Omega_{2}\left(\phi_{M}\right)$ are positive definite. Then, for each $i \in \mathcal{M}$, define

$$
\beta_{i} \triangleq-\frac{1}{2} \operatorname{vec}^{-1}\left[\Omega_{2}^{-1}\left(\phi_{i}\right) \Omega_{1}\left(\phi_{i}\right)\right] \in \mathbb{R}^{b \times l},
$$

and it follows from Proposition 2 that $\beta_{i}$ is the unique global minimizer of $\mathcal{J}_{i}$. Next, let $\ell \in \mathcal{M}$ be the smallest integer such that $\mathcal{J}_{\ell}\left(\beta_{\ell}\right)=\min _{i \in \mathcal{M}} \mathcal{J}_{i}\left(\beta_{i}\right)$. Thus, the identified parameters are $\beta^{+} \triangleq \beta_{\ell}$ and $\phi^{+} \triangleq \phi_{\ell}$, and the identified transfer function matrices are

$$
\begin{aligned}
& G_{\mathrm{ff}}^{+}(z) \triangleq \mathcal{G}_{\mathrm{ff}}\left(z, \beta^{+}\right)=z^{-n_{\mathrm{ff}}} \mathcal{N}_{\mathrm{ff}}\left(z, \beta^{+}\right), \\
& G_{\mathrm{fb}}^{+}(z) \triangleq \mathcal{G}_{\mathrm{fb}}\left(z, \phi^{+}\right)=\mathcal{D}_{\mathrm{fb}}^{-1}\left(z, \phi^{+}\right) \mathcal{N}_{\mathrm{fb}}\left(z, \phi^{+}\right) .
\end{aligned}
$$

Note that $\arg \min _{i \in \mathcal{M}} \mathcal{J}_{i}\left(\beta_{i}\right)$ is not necessarily unique. In this case, $\ell \in \mathcal{M}$ is the smallest integer such that $\mathcal{J}_{\ell}\left(\beta_{\ell}\right)=\min _{i \in \mathcal{M}} \mathcal{J}_{i}\left(\beta_{i}\right)$. In practice, $\arg \min _{i \in \mathcal{M}} \mathcal{J}_{i}\left(\beta_{i}\right)$ is generally unique. We now summarize this SSID method.

Algorithm 1 Consider the closed-loop transfer function matrix (4), where $G_{y}, G_{v}$, and $\left\{H\left(\theta_{k}\right)\right\}_{k=1}^{N}$ are known, and (A1)-(A3) are satisfied. Then, the subsystem identification algorithm is as follows:

Step 1. Generate the candidate pool $\Phi \subseteq \mathcal{S}$ and candidate sequence $\left\{\phi_{i}\right\}_{i=1}^{M}$

Step 2. For each $i \in \mathcal{M}$, find the global minimizer of $\mathcal{J}_{i}$, which is $\beta_{i}=-\frac{1}{2} \operatorname{vec}^{-1}\left[\Omega_{2}^{-1}\left(\phi_{i}\right) \Omega_{1}\left(\phi_{i}\right)\right] \in \mathbb{R}^{b \times l}$.

Step 3. Find the smallest integer $\ell \in \mathcal{M}$ such that $\partial_{\ell}\left(\beta_{\ell}\right)=\min _{i \in \mathcal{M}} \partial_{i}\left(\beta_{i}\right)$.
Step 4. The identified parameters are $\beta^{+}=\beta_{\ell}$ and $\phi^{+}=\phi_{\ell}$, and the identified transfer function matrices are $G_{\mathrm{ff}}^{+}(z)=\mathcal{G}_{\mathrm{ff}}\left(z, \beta^{+}\right)$, and $G_{\mathrm{fb}}^{+}(z)=\mathcal{G}_{\mathrm{fb}}\left(z, \phi^{+}\right)$.

\section{Analysis of Algorithm 1}

We assume $N_{\mathrm{fb}}$ and $D_{\mathrm{fb}}$ are left coprime, and impose the following assumption, which is stronger than (A2):

(A4) $N>n_{y}+(2 m-1)\left(d+d_{\mathrm{fb}}\right)+n_{\mathrm{ff}}+\max \left\{d_{\mathrm{fb}}, n_{\mathrm{fb}}\right\}$.

Assumption (A4) requires that the number $N$ of frequency response data points is sufficiently large. This assumption is used in the next result to obtain sufficient conditions on $\beta \in \mathbb{R}^{b \times l}$ and $\phi \in \mathbb{R}^{a \times m}$ such that $\tilde{\mathcal{G}}(z, \beta, \phi) \equiv \tilde{G}(z)$. The proof is in Appendix A.

Proposition 3 Let $\beta \in \mathbb{R}^{b \times l}$ and $\phi \in \mathbb{R}^{a \times m}$, and assume (A4) is satisfied. Then, $\sum_{k=1}^{N} \| \tilde{\mathcal{G}}\left(\sigma_{k}, \beta, \phi\right)-$ $\tilde{G}\left(\sigma_{k}\right) \|_{\mathrm{F}}=0$ if and only if $\tilde{\mathcal{G}}(z, \beta, \phi) \equiv \tilde{G}(z)$.

The conditions in Proposition 3 are not sufficient to conclude that $\beta=\beta_{*}$ and $\phi=\phi_{*}$. The following example demonstrates this scenario.

Example 1 Let

$$
\begin{gathered}
G_{y}(z)=G_{v}(z)=\frac{1}{z-0.7} \\
G_{\mathrm{ff}}(z)=-0.4, \quad G_{\mathrm{fb}}(z)=\frac{1.2 z+0.2}{z-1},
\end{gathered}
$$

and note that $\beta_{*}=-0.4$ and $\phi_{*}=\left[\begin{array}{lll}1.2 & 0.2 & -1\end{array}\right]^{\mathrm{T}}$. The closed-loop transfer function $(4)$ is $\tilde{G}(z)=(0.8 z+$ $0.6) /\left(z^{2}-0.5 z+0.9\right)$. Let $\beta=0.3 \neq \beta_{*}$ and $\phi=$ $\left[\begin{array}{lll}0.5 & 0.69-0.3\end{array}\right]^{\mathrm{T}} \neq \phi_{*}$, and it follows that $\tilde{\mathcal{G}}(z, \beta, \phi) \equiv$ $\tilde{G}(z)$.

In Example 1, the SSID problem is not well posed, because $\left(\beta_{*}, \phi_{*}\right)$ cannot be uniquely determined from the noiseless frequency response data. See [4, Chap. 13] for more details in the case with feedback only. We now impose an additional assumption to ensure that $\tilde{\mathcal{G}}(z, \beta, \phi) \equiv \tilde{G}(z)$ if and only if $\beta=\beta_{*}$ and $\phi=\phi_{*}$.

Let $\Psi \subseteq \mathbb{R}^{a \times m}$ be a compact set with no isolated points such that $\phi_{*} \in \Psi$. In practice, $\Psi$ is used to generate the candidate pool. We assume $\Psi$ is known, and

(A5) If $\beta \in \mathbb{R}^{b \times l}, \phi \in \Psi \cap \mathcal{S}$, and $\tilde{\mathcal{G}}(z, \beta, \phi) \equiv \tilde{G}(z)$, then $\beta=\beta_{*}$ and $\phi=\phi_{*}$.

Example 2 Consider $G_{y}, G_{v}, G_{\mathrm{ff}}$, and $G_{\mathrm{fb}}$ given by (15) and (16), and note that $\beta_{*}=-0.4$ and $\phi_{*}=$ $\left[\begin{array}{lll}1.2 & 0.2 & -1\end{array}\right]^{\mathrm{T}}$. Example 1 shows that $(\beta, \phi)=\left(\beta_{*}, \phi_{*}\right)$ is not the only point in $\mathbb{R} \times \mathcal{S}$ such that $\tilde{\mathcal{G}}(z, \beta, \phi) \equiv \tilde{G}(z)$. We now define $\Psi \triangleq[0,2] \times[0,2] \times[-1.5,-0.5]$, and note that $\phi_{*} \in \Psi$. Furthermore, it can be confirmed that $(\beta, \phi)=\left(\beta_{*}, \phi_{*}\right)$ is the only point in $\mathbb{R} \times(\mathcal{S} \cap \Psi)$ such that $\tilde{\mathcal{G}}(z, \beta, \phi) \equiv \tilde{G}(z)$. Therefore, (A5) is satisfied.

The following result addresses the case where $\phi_{*}$ is in the candidate pool $\Phi$. This result demonstrates that a 
sufficiently small noise $\left\|\eta_{*}\right\|$ yields identified parameters $\beta^{+}$and $\phi^{+}$such that $\phi^{+}=\phi_{*}$ and $\beta^{+}$is arbitrarily close to $\beta_{*}$. The proof is in Appendix B.

Theorem 1 Assume (A1)-(A5) are satisfied. Let $\Phi \subseteq$ $(\Psi \cap \mathcal{S})$, and assume $\phi_{*} \in \Phi$. Let $\beta^{+}$and $\phi^{+}$denote the identified parameters obtained from Algorithm 1 with the candidate pool $\Phi$. Then, the following statements hold:

(i) There exists $\delta_{0}>0$ such that if $\left\|\eta_{*}\right\|<\delta_{0}$, then $\phi^{+}=\phi_{*}$. Moreover, for all $\epsilon>0$, there exists $\delta \epsilon$ $\left(0, \delta_{0}\right)$ such that if $\left\|\eta_{*}\right\|<\delta$, then $\beta^{+} \in \mathbb{B}_{\epsilon}\left(\beta_{*}\right)$.

(ii) If $\eta_{*}=0$, then $\beta^{+}=\beta_{*}$ and $\phi^{+}=\phi_{*}$.

We now extend the analysis to address the case where $\phi_{*}$ is not necessarily in the candidate pool $\Phi$. Let $\rho \in$ $(0,1)$ be such that if $\lambda \in \mathbb{C}$ and $\operatorname{det} \tilde{\mathcal{D}}\left(\lambda, \phi_{*}\right)=0$, then $|\lambda|<\rho$, and define

$$
\begin{gathered}
\mathcal{S}_{\rho} \triangleq\left\{\phi \in \mathbb{R}^{a \times m}: \text { if } \lambda \in \mathbb{C} \text { and } \operatorname{det} \tilde{\mathcal{D}}(\lambda, \phi)=0,\right. \\
\text { then }|\lambda|<\rho\} .
\end{gathered}
$$

Note that as $\rho$ approaches $1, \mathcal{S} \backslash \mathcal{S}_{\rho}$ approaches the empty set. In practice, $\mathcal{S}_{\rho}$ is used to generate the candidate pool, and $\rho$ can be selected sufficiently closed to 1 to ensure that $\phi_{*} \in \mathcal{S}_{\rho}$.

In the following result, we consider Algorithm 1 with a sequence of candidate pools that converge to $\Psi \cap \mathcal{S}_{\rho}$, which is bounded and contains no isolated points $[17$, Prop. 7]. This result demonstrates that a sufficiently dense candidate pool and sufficiently small noise $\left\|\eta_{*}\right\|$ yield identified parameters $\beta^{+}$and $\phi^{+}$that are arbitrarily close to $\beta_{*}$ and $\phi_{*}$. The proof is in Appendix B.

Theorem 2 Assume (A1)-(A5) are satisfied. For all $j \in \mathbb{Z}^{+}$, let $\Lambda_{j} \subseteq\left(\Psi \cap \mathcal{S}_{\rho}\right)$ be a finite set such that $\left\{\Lambda_{j}\right\}_{j=1}^{\infty}$ converges to $\Psi \cap \mathcal{S}_{\rho}$. For each $j \in \mathbb{Z}^{+}$, let $\beta_{j}^{+}$ and $\phi_{j}^{+}$denote the identified parameters obtained from Algorithm 1 with the candidate pool $\Phi=\Lambda_{j}$. Then, for all $\epsilon>0$, there exist $\delta>0$ and $L \in \mathbb{Z}^{+}$such that if $\left\|\eta_{*}\right\|<\delta$ and $j>L$, then $\beta_{j}^{+} \in \mathbb{B}_{\epsilon}\left(\beta_{*}\right)$ and $\phi_{j}^{+} \in \mathbb{B}_{\epsilon}\left(\phi_{*}\right)$.

\section{$6 \quad$ Numerical Examples}

We present examples, where $m=l=n=2$. For all examples, let

$$
\begin{gathered}
D(z)=\operatorname{diag}(z+0.1, z-0.2), \quad D_{\mathrm{fb}}(z)=I_{2} \\
N_{y}(z)=N_{v}(z)=\operatorname{diag}(1,-1),
\end{gathered}
$$

and

$N_{\mathrm{fb}}(z)=\left[\begin{array}{cc}0.3 & 1 \\ 0.2 & 0.4\end{array}\right], \quad N_{\mathrm{ff}}(z)=\left[\begin{array}{cc}z-0.3 & z+0.4 \\ 0.5 z & 1\end{array}\right]$.

Note that

$$
\beta_{*}=\left[\begin{array}{cccc}
1 & 0.5 & -0.3 & 0 \\
1 & 0 & 0.4 & 1
\end{array}\right]^{\mathrm{T}}, \quad \phi_{*}=\left[\begin{array}{cc}
0.3 & 1 \\
0.2 & 0.4
\end{array}\right]^{\mathrm{T}}
$$

Let $N=20$, and for $k \in \mathcal{N}$, let $\theta_{k}=0.02 \pi k$. This example satisfies (A1)-(A4), and for any compact set
$\Psi \subseteq \mathbb{R}^{a \times m}$ containing $\phi_{*},(\mathrm{~A} 5)$ is satisfied.

Example 3 Consider the case with $\phi_{*} \in \Phi$ and noiseless data. Define the candidate pool $\Lambda_{0} \triangleq\{\phi \in$ $\mathbb{R}^{2 \times 2}:$ for $\left.i, j \in\{1,2\}, \phi_{(i, j)} \in\{-0.5+0.1 k\}_{k=0}^{20}\right\} \cap \mathcal{S}$, and note that $\phi_{*} \in \Lambda_{0}$. Algorithm 1 is used with the candidate pool $\Phi=\Lambda_{0}$ to obtain $\beta^{+}=\beta_{*}$ and $\phi^{+}=\phi_{*}$, which agrees with (ii) of Theorem 1.

Example 4 Consider the case with $\phi_{*} \in \Phi$ and noisy data. For $i=1, \ldots, 15$, let $\Gamma_{i}(z) \in \mathbb{C}^{2 \times 2}$ be the noise, and define the noise-to-signal ratio $R_{i} \triangleq \frac{1}{N} \sum_{k=1}^{N}\left\|\Gamma_{i}\left(\sigma_{k}\right)\right\|_{\mathrm{F}} /\left\|\tilde{G}\left(\sigma_{k}\right)\right\|_{\mathrm{F}}$. For $i=1, \ldots, 15$, the frequency response data is $H_{i}\left(\theta_{k}\right) \triangleq \tilde{G}\left(\sigma_{k}\right)+\Gamma_{i}\left(\sigma_{k}\right)$. In this example, $\Gamma_{1}, \ldots, \Gamma_{15}$ are randomly generated such that $R_{1}>R_{2}>\cdots>R_{15}$. Specifically, $R_{1}=2.39, R_{2}=1.29, R_{4}=0.28, R_{7}=3.76 \times 10^{-2}$, and $R_{15}=1.42 \times 10^{-4}$. For $i=1, \ldots, 15$, Algorithm 1 is used with the candidate pool $\Phi=\Lambda_{0}$ and data $\left\{H_{i}\left(\theta_{k}\right)\right\}_{k=1}^{N}$ to obtain the identified parameters $\beta_{i}^{+}$ and $\phi_{i}^{+}$. Figure 3 shows that for $i \geq 7, \phi_{i}^{+}=\phi_{*}$, and for sufficiently large $i,\left\|\beta_{i}^{+}-\beta_{*}\right\|_{\mathrm{F}}$ is arbitrarily small, which agrees with (i) of Theorem 1 .

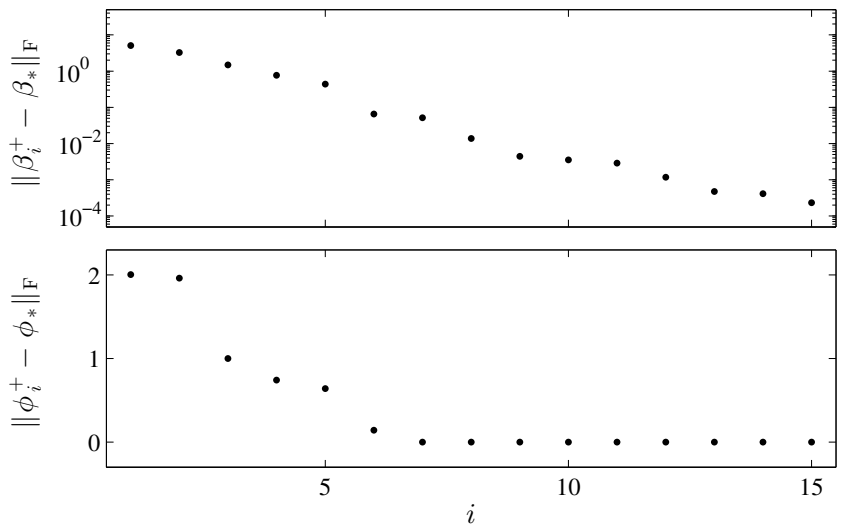

Fig. 3. Noisy data and $\phi_{*} \in \Phi$. For $i=1, \ldots, 15$, Algorithm 1 is used with the candidate pool $\Lambda_{0}$ and data $\left\{H_{i}\left(\theta_{k}\right)\right\}_{k=1}^{N}$ to obtain $\beta_{i}^{+}$and $\phi_{i}^{+}$. For $i \geq 7, \phi_{i}^{+}=\phi_{*}$, and for sufficiently large $i,\left\|\beta_{i}^{+}-\beta_{*}\right\|_{\mathrm{F}}$ is arbitrarily small.

Example 5 Consider the case with $\phi_{*} \notin \Phi$ and noisy data. For $j=1, \ldots, 18$, define the candidate pool $\Lambda_{j} \triangleq$ $\left\{\phi \in \mathbb{R}^{2 \times 2}:\right.$ for $i, h \in\{1,2\}, \phi_{(i, h)} \in\{-0.5+2 k /(1+$ $\left.j)\}_{k=0}^{1+j}\right\} \cap \mathcal{S}_{\rho}$, where $\rho=0.99$, and note that for $j=$ $1, \ldots, 18, \phi_{*} \notin \Lambda_{j}$. For $i=1, \ldots, 15$ and $j=1, \ldots, 18$, Algorithm 1 is used with the candidate pool $\Phi=\Lambda_{j}$ and data $\left\{H_{i}\left(\theta_{k}\right)\right\}_{k=1}^{N}$ to obtain the identified parameters $\beta_{j, i}^{+}$and $\phi_{j, i}^{+}$. Figure 4 shows that for sufficient large $j$ and $i,\left\|\beta_{j, i}^{+}-\beta_{*}\right\|_{\mathrm{F}}$ and $\left\|\phi_{j, i}^{+}-\phi_{*}\right\|_{\mathrm{F}}$ are arbitrarily small, which agrees with Theorem 2 .

\section{Computational Complexity}

The computational complexity of Algorithm 1 is dominated by Step 2, where a quadratic minimization problem is solved by $M$ times. We use the modified Gram Schmidt method [22, Chap. 14] to perform this 

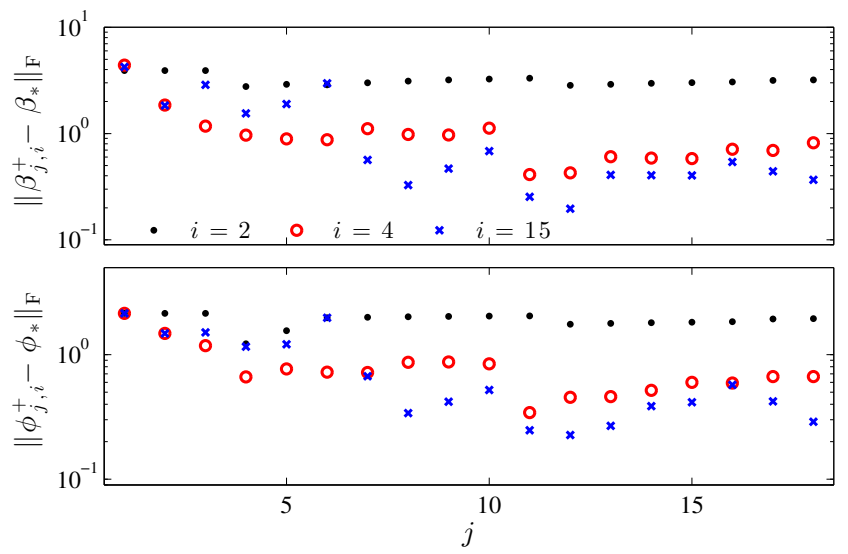

Fig. 4. Noisy data and $\phi_{*} \notin \Phi$. For $i=2,4,15$, and $j=1, \ldots, 18$, Algorithm 1 is used with the candidate pool $\Lambda_{j}$ and data $\left\{H_{i}\left(\theta_{k}\right)\right\}_{k=1}^{N}$ to obtain $\beta_{j, i}^{+}$and $\phi_{j, i}^{+}$. For sufficient large $j$ and $i,\left\|\beta_{j, i}^{+}-\beta_{*}\right\|_{\mathrm{F}}$ and $\left\|\phi_{j, i}^{+}-\phi_{*}\right\|_{\mathrm{F}}$ are arbitrarily small.

quadratic minimization. It follows from [22, Chap. 14] that the computational complexity of Algorithm 1 is $C \triangleq M\left[4 N n m^{2} l^{3}\left(n_{\mathrm{ff}}+1\right)^{2}+2 N n m l^{2}\left(n_{\mathrm{ff}}+1\right)\right]$ flops.

We compare the computational complexity of Algorithm 1 with the SSID algorithm in [17]. The SSID method in [17] also uses a candidate pool approach; however, $\mathcal{G}_{\mathrm{ff}}$ is parameterized as an IIR transfer function, and the method only applies to subsystems that are SISO (i.e., $v=y$ and $l=m=n=1$ ). For SISO subsystems, the computational complexity of Algorithm 1 is $C=M\left[4 N\left(n_{\mathrm{ff}}+1\right)^{2}+2 N\left(n_{\mathrm{ff}}+1\right)\right]$ flops.

Next, consider [17, Alg. 1], where $\hat{N}$ denotes the number of frequency response data points, $M_{\mathrm{fb}}$ denotes the number of elements in the feedback candidate pool, and $M_{\mathrm{ff}}$ denotes the number of elements in the feedforward candidate pool. Let $\hat{n}_{\mathrm{ff}}$ denote the degree of numerator of $\mathcal{G}_{\mathrm{ff}}$. The computational complexity of $[17, \mathrm{Alg} .1]$ is $\hat{C}=M_{\mathrm{ff}} M_{\mathrm{fb}}\left[4 \hat{N}\left(\hat{n}_{\mathrm{ff}}+1\right)^{2}+2 \hat{N}\left(\hat{n}_{\mathrm{ff}}+1\right)\right]$ flops.

To compare the computational complexities of these algorithms, we assume these algorithms use the same frequency response data and feedback candidate pool, which implies that $N=\hat{N}$ and $M=M_{\mathrm{fb}}$. Thus, the computational complexity ratio is

$$
\mathcal{R} \triangleq \frac{C}{\hat{C}}=\frac{2\left(n_{\mathrm{ff}}+1\right)^{2}+\left(n_{\mathrm{ff}}+1\right)}{M_{\mathrm{ff}}\left[2\left(\hat{n}_{\mathrm{ff}}+1\right)^{2}+\left(\hat{n}_{\mathrm{ff}}+1\right)\right]} .
$$

In general, $\hat{n}_{\mathrm{ff}} \leq n_{\mathrm{ff}}$ and $M_{\mathrm{ff}} \gg 1$, which result $\mathcal{R}<1$.

\section{Example 6 Let}

$$
\begin{gathered}
G_{y}(z)=G_{v}(z)=\frac{0.4}{z-0.9}, \\
G_{\mathrm{ff}}(z)=\frac{0.2(z-0.77)}{(z-0.83)(z-0.89)}, \quad G_{\mathrm{fb}}(z)=\frac{0.32}{z-0.81} .
\end{gathered}
$$

Let $N=31$, and for $k \in \mathcal{N}$, let $\theta_{k}=0.008 \pi(k-1)$. Consider the candidate pool $\Lambda_{0} \triangleq\left\{\phi \in \mathbb{R}^{2}: \phi_{(1)} \in\right.$ $\left.\{-2+0.05 k\}_{k=0}^{40}, \phi_{(2)} \in\{-2+0.05 k\}_{k=0}^{80}\right\} \cap \mathcal{S}$, which contains 297 elements.

First, we use Algorithm 1 with the candidate pool $\Lambda_{0}$. For $n_{\mathrm{ff}}=1,2, \ldots, 17$, we identify feedforward and feedback controllers, where $\mathcal{G}_{\text {ff }}$ is FIR even though $G_{\mathrm{ff}}$ is IIR.

Next, consider [17, Alg. 1], where $\mathcal{G}_{\text {ff }}$ is parameterized as IIR with $\hat{n}_{\mathrm{ff}}=1$. Consider feedforward candidate pool $F=\left\{\left[\begin{array}{ll}x_{1}+x_{2} & x_{1} x_{2}\end{array}\right]^{\mathrm{T}} \in \mathbb{R}^{2}: x_{1}, x_{2} \in\{-0.05 k\}_{k=0}^{19}\right\}$, which contains $M_{\mathrm{ff}}=210$ elements. We use [17, Alg. 1] with the candidate pool $F \times \Lambda_{0}$ to identify feedback and feedforward controllers.

Figure 5 shows the identification errors

$$
\begin{gathered}
\mathcal{E}_{\mathrm{fb}} \triangleq \int_{\theta_{1}}^{\theta_{N}}\left|G_{\mathrm{fb}}^{+}\left(e^{\jmath \theta}\right)-G_{\mathrm{fb}}\left(e^{\jmath \theta}\right)\right| \mathrm{d} \theta, \\
\mathcal{E}_{\mathrm{ff}} \triangleq \int_{\theta_{1}}^{\theta_{N}}\left|G_{\mathrm{ff}}^{+}\left(e^{\jmath \theta}\right)-G_{\mathrm{ff}}\left(e^{\jmath \theta}\right)\right| \mathrm{d} \theta,
\end{gathered}
$$

for each algorithm. In this example, for $n_{\mathrm{ff}}=14, \ldots, 17$, Algorithm 1 yields errors $\mathcal{E}_{\mathrm{fb}}$ and $\mathcal{E}_{\mathrm{ff}}$ that are comparable to those obtained from [17, Alg. 1]. Figure 5 also shows that for $n_{\mathrm{ff}}=1,2, \ldots, 17, \mathcal{R}<0.32$.

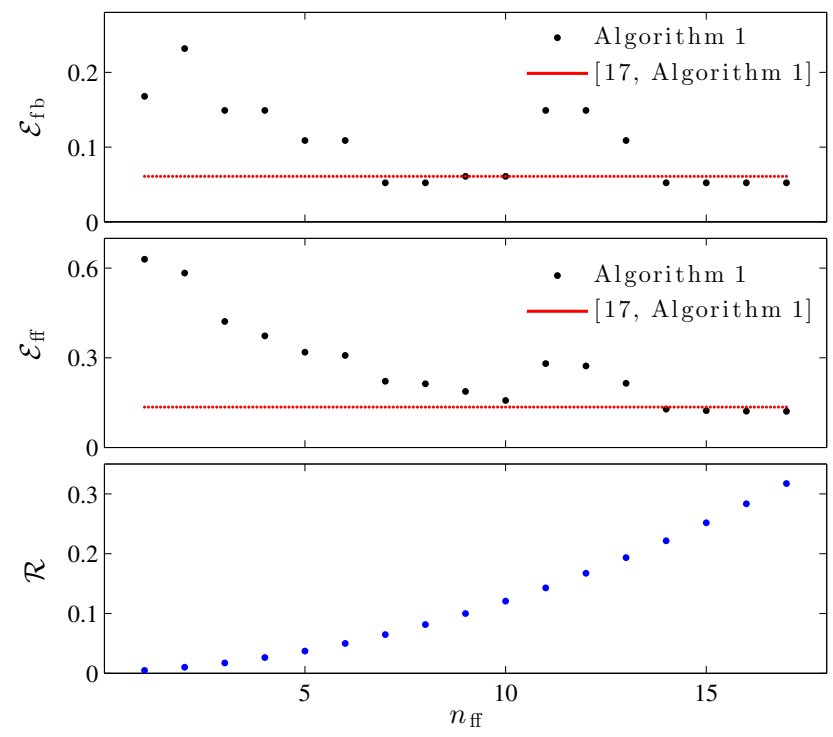

Fig. 5. Computation complexity. For $n_{\mathrm{ff}}=14, \ldots, 17$, Algorithm 1 yields errors $\mathcal{E}_{\mathrm{fb}}$ and $\mathcal{E}_{\mathrm{ff}}$ that are comparable to those obtained from [17, Alg. 1]. For $n_{\mathrm{ff}}=1,2, \ldots, 17, \mathcal{R}<0.32$.

\section{Conclusion}

We presented a discrete-time SSID algorithm for identifying MIMO feedback and feedforward subsystems. This SSID algorithm uses closed-loop input-output data but does not rely on the measurement of any internal signals. The method ensures asymptotic stability of the identified closed-loop transfer function matrix. The analytic results are Theorems 1 and 2, which provide properties of the SSID algorithm. In particular, Theorem 2 shows that the parameters of the identified feedback and feedforward transfer function matrices are arbitrar- 
ily close to the true parameters if the candidate pool is sufficiently dense and the data noise is sufficiently small.

\section{Appendix A Proofs of Propositions 1 and 3}

Proof of Proposition 1. Let $\phi \in \mathcal{S}$. Then, for all $k \in \mathcal{N}, \tilde{\mathcal{D}}\left(\sigma_{k}, \phi\right)$ is nonsingular, and thus, it follows from (9) and (14) that $\Omega_{2}(\phi)$ is well defined and positive semidefinite. Assume for contradiction that there exists $x \in \mathbb{R}^{b} \backslash\{0\}$ such that $x^{\mathrm{T}}\left[\operatorname{Re} \sum_{k=1}^{N} \mathcal{A}_{k}^{*}(\phi) \mathcal{A}_{k}(\phi)\right] x=0$. Let $k \in \mathcal{N}$, and it follows that $\mathcal{A}_{k}(\phi) x=0$. Define $\Xi(z) \triangleq N_{y}(z) \tilde{\mathcal{D}}^{\mathrm{A}}(z, \phi) \mathcal{D}_{\mathrm{fb}}(z, \phi) \nu(z) x \in \mathbb{R}^{n}[z]$. Thus, $(9)$ implies that $0=\Xi\left(\sigma_{k}\right) /\left[\sigma_{k}^{n_{\mathrm{ff}}} \operatorname{det} \tilde{\mathcal{D}}\left(\sigma_{k}, \phi\right)\right]$, which implies that $\Xi\left(\sigma_{k}\right)=0$. Since $\operatorname{deg} \tilde{\mathcal{D}}=d+d_{\mathrm{fb}}$, it follows that $\operatorname{deg} \tilde{\mathcal{D}}^{\mathrm{A}} \leq(m-1)\left(d+d_{\mathrm{fb}}\right)$. Since, in addition, $\operatorname{deg} \nu(z) x \leq n_{\mathrm{ff}}$, it follows from (A2) that $\operatorname{deg} \Xi \leq$ $n_{y}+(m-1)\left(d+d_{\mathrm{fb}}\right)+d_{\mathrm{fb}}+n_{\mathrm{ff}}<N$. Since $\Xi\left(\sigma_{1}\right)=$ $\cdots=\Xi\left(\sigma_{N}\right)=0$ and $\operatorname{deg} \Xi<N$, it follows that $\Xi=0$.

Define $\mathcal{T} \triangleq\left\{z \in \mathbb{C}: \min \left\{\operatorname{rank} N_{y}(z), \operatorname{rank} \tilde{\mathcal{D}}^{\mathrm{A}}(z, \phi)\right.\right.$, $\left.\left.\operatorname{rank} \mathcal{D}_{\mathrm{fb}}(z, \phi)\right\}<m\right\}$. Since, for all $z \in \mathbb{C} \backslash \mathcal{T}$, $\operatorname{rank} N_{y}(z)=m \leq n$, it follows that $N_{y}$ has full column rank, which implies that for all $z \in \mathbb{C} \backslash \mathcal{T}$, $N_{y}(z)$ is left invertible. Thus, for all $z \in \mathbb{C} \backslash \mathcal{T}$, $\tilde{\mathcal{D}}^{\mathrm{A}}(z, \phi) \mathcal{D}_{\mathrm{fb}}(z, \phi) \nu(z) x=0$. Since for all $z \in \mathbb{C} \backslash \mathcal{T}$, $\tilde{\mathcal{D}}^{\mathrm{A}}(z, \phi)$ and $\mathcal{D}_{\mathrm{fb}}(z, \phi)$ are nonsingular, it follows that for all $z \in \mathbb{C} \backslash \mathcal{T}, \nu(z) x=0$. Finally, the structure of $\nu$ implies that $x=0$, which is a contradiction. Thus, $\operatorname{Re} \sum_{k=1}^{N} \mathcal{A}_{k}^{*}(\phi) \mathcal{A}_{k}(\phi)$ is positive definite. Therefore, it follows from (14) that $\Omega_{2}(\phi)$ is positive definite.

Proof of Proposition 3. Let $\beta \in \mathbb{R}^{b \times l}$ and $\phi \in \mathbb{R}^{m \times a}$, and define $\mathcal{O}: \mathbb{C} \rightarrow \mathbb{C}^{n \times l}$ by $\mathcal{O}(z) \triangleq \tilde{\mathcal{G}}(z, \beta, \phi)-\tilde{G}(z)$. Next, define $\mathcal{P}(z) \triangleq N_{y}(z) \tilde{\mathcal{D}}^{\mathrm{A}}(z, \phi)\left[z^{n_{\mathrm{ff}}} \mathcal{N}_{\mathrm{fb}}(z, \phi)+\right.$ $\left.\mathcal{D}_{\mathrm{fb}}(z, \phi) \mathcal{N}_{\mathrm{ff}}(z, \beta)\right] \in \mathbb{R}^{n \times l}[z], \mathcal{Q}(z) \triangleq N_{y}(z) \tilde{\mathcal{D}}^{\mathrm{A}}\left(z, \phi_{*}\right) \times$ $\left[z^{n_{\mathrm{ff}}} \mathcal{N}_{\mathrm{fb}}\left(z, \phi_{*}\right)+\mathcal{D}_{\mathrm{fb}}\left(z, \phi_{*}\right) \mathcal{N}_{\mathrm{ff}}\left(z, \beta_{*}\right)\right] \in \mathbb{R}^{n \times l}[z]$, and $\mathcal{H}(z) \triangleq \mathcal{P}(z) \operatorname{diag} \tilde{\mathcal{D}}\left(z, \phi_{*}\right)-\mathcal{Q}(z) \operatorname{diag} \tilde{\mathcal{D}}(z, \phi) \in \mathbb{R}^{n \times l}[z]$. Note that $\mathcal{O}(z)=\mathcal{H}(z) /\left[z^{n_{\mathrm{ff}}} \operatorname{det} \tilde{\mathcal{D}}(z, \phi) \operatorname{det} \tilde{\mathcal{D}}\left(z, \phi_{*}\right)\right]$. Since $\sum_{k=1}^{N}\left\|\mathcal{O}\left(\sigma_{k}\right)\right\|_{\mathrm{F}}=0$, it follows that for all $k \in \mathcal{N}$, $\mathcal{O}\left(\sigma_{k}\right)=0$, which implies that $\mathcal{H}\left(\sigma_{k}\right)=0$. Since $\operatorname{deg} \operatorname{det} \tilde{\mathcal{D}}(z, \phi)=\operatorname{deg} \operatorname{det} \tilde{\mathcal{D}}\left(z, \phi_{*}\right)=m\left(d+d_{\mathrm{fb}}\right)$ and $\operatorname{deg} \mathcal{P}, \operatorname{deg} \mathcal{Q} \leq n_{\mathrm{ff}}+n_{y}+(m-1)\left(d+d_{\mathrm{fb}}\right)+\max \left\{d_{\mathrm{fb}}, n_{\mathrm{fb}}\right\}$, it follows that $\operatorname{deg} \mathcal{H} \leq n_{y}+(2 m-1)\left(d+d_{\mathrm{fb}}\right)+n_{\mathrm{ff}}+$ $\max \left\{d_{\mathrm{fb}}, n_{\mathrm{fb}}\right\}$. Since for all $k \in \mathcal{N}, \mathcal{H}\left(\sigma_{k}\right)=0$, and $(\mathrm{A} 4)$ implies that $\operatorname{deg} \mathcal{H}<N$, it follows that $\mathcal{H}=0$, which implies that $\mathcal{O}=0$. Thus, $\tilde{\mathcal{G}}(z, \beta, \phi) \equiv \tilde{G}(z)$.

\section{Appendix B Proofs of Theorems 1 and 2}

The following notation is needed in the proofs of Theorems 1 and 2. Define $\hat{\Omega}_{1}: \mathcal{S} \times \mathbb{C}^{n \times l N} \rightarrow \mathbb{R}^{l b}$ by $\hat{\Omega}_{1}(\phi, \eta) \triangleq$ $\Omega_{1}(\phi)+2 \operatorname{Re} \sum_{k=1}^{N} \operatorname{vec} \mathcal{A}_{k}^{*}(\phi)\left[H\left(\theta_{k}\right)-\tilde{G}\left(\sigma_{k}\right)-\eta_{k}\right]$, where $\eta_{1}, \ldots, \eta_{N} \in \mathbb{C}^{n \times l}$ and $\eta \triangleq\left[\eta_{1} \cdots \eta_{N}\right] \in \mathbb{C}^{n \times l N}$. Note that $\hat{\Omega}_{1}\left(\phi, \eta_{*}\right)=\Omega_{1}(\phi)$. Thus, $\hat{\Omega}_{1}$ is a function not only of $\phi$ but also the noise $\eta$. Define $\hat{J}: \mathbb{R}^{b \times l} \times \mathcal{S} \times \mathbb{C}^{n \times l N} \rightarrow$ $[0, \infty), \hat{\theta}: \mathcal{S} \times \mathbb{C}^{n \times l N} \rightarrow \mathbb{R}^{b \times l}$, and $\hat{Q}: \mathcal{S} \times \mathbb{C}^{n \times l N} \rightarrow$
$[0, \infty)$ by

$$
\begin{aligned}
\hat{J}(\beta, \phi, \eta) & \triangleq \sum_{k=1}^{N}\left\|\tilde{\mathcal{G}}\left(\sigma_{k}, \beta, \phi\right)-\tilde{G}\left(\sigma_{k}\right)-\eta_{k}\right\|_{\mathrm{F}}^{2} \in \mathbb{R}, \\
\hat{\theta}(\phi, \eta) & \triangleq-\frac{1}{2} \operatorname{vec}^{-1}\left[\Omega_{2}^{-1}(\phi) \hat{\Omega}_{1}(\phi, \eta)\right] \in \mathbb{R}^{b \times l} \\
\hat{Q}(\phi, \eta) & \triangleq \hat{J}(\hat{\theta}(\phi, \eta), \phi, \eta) \in \mathbb{R} .
\end{aligned}
$$

Note that $\hat{J}\left(\beta, \phi, \eta_{*}\right)=J(\beta, \phi)$.

It follows from $(6),(9),(10)$, and (17)-(19) that

$$
\begin{aligned}
\hat{\theta}\left(\phi_{*}, 0\right) & =-\frac{1}{2} \operatorname{vec}^{-1}\left[\Omega_{2}^{-1}\left(\phi_{*}\right) \hat{\Omega}_{1}\left(\phi_{*}, 0\right)\right]=\beta_{*}, \\
\hat{Q}\left(\phi_{*}, 0\right) & =\hat{J}\left(\beta_{*}, \phi_{*}, 0\right)=0 .
\end{aligned}
$$

Proof of Theorem 1. To prove (i), let $\phi \in \Phi \backslash\left\{\phi_{*}\right\}$, it follows from (19), Proposition 3, and (A5) that $\hat{Q}(\phi, 0)>0$. Define $U \triangleq \min _{x \in \Phi \backslash\left\{\phi_{*}\right\}} \hat{Q}(x, 0)>0$. It can be shown that $\hat{Q}$ is continuous on $\mathcal{S} \times \mathbb{C}^{n \times l N}$. Thus, for each $j \in \mathcal{M}, \hat{Q}\left(\phi_{j}, \cdot\right)$ is continuous on $\mathbb{C}^{n \times l N}$, which implies that, for each $j \in \mathcal{M}$, there exists $\delta_{j}>0$ such that for all $\eta \in \mathbb{B}_{\delta_{j}}(0),\left|\hat{Q}\left(\phi_{j}, \eta\right)-\hat{Q}\left(\phi_{j}, 0\right)\right|<U / 2$. Define $\delta_{0} \triangleq \min _{j \in \mathcal{M}} \delta_{j}$, and assume that $\left\|\eta_{*}\right\|<\delta_{0}$. Since $\phi_{*} \in \Phi$, it follows that there exists $i \in \mathcal{M}$ such that $\phi_{i}=\phi_{*}$. Since $\hat{Q}\left(\phi_{i}, 0\right)=\hat{Q}\left(\phi_{*}, 0\right)=0$, it follows that $\hat{Q}\left(\phi_{i}, \eta_{*}\right)=\left|\hat{Q}\left(\phi_{i}, \eta_{*}\right)-\hat{Q}\left(\phi_{i}, 0\right)\right|<U / 2$. Let $j \in \mathcal{M} \backslash\{i\}$, and it follows that $-U / 2<\hat{Q}\left(\phi_{j}, \eta_{*}\right)-\hat{Q}\left(\phi_{j}, 0\right)$, which implies that $\hat{Q}\left(\phi_{j}, \eta_{*}\right)>\hat{Q}\left(\phi_{j}, 0\right)-U / 2$. Since, in addition, $\hat{Q}\left(\phi_{j}, 0\right) \geq U$, it follows that $\hat{Q}\left(\phi_{j}, \eta_{*}\right)>U / 2$. Thus, $\hat{Q}\left(\phi_{i}, \eta_{*}\right)<\hat{Q}\left(\phi_{j}, \eta_{*}\right)$, which implies that $\mathcal{J}_{i}\left(\beta_{i}\right)<\mathcal{J}_{j}\left(\beta_{j}\right)$ using (8) and (17)-(19). Therefore, it follows from Algorithm 1 that $\phi^{+}=\phi_{i}=\phi_{*}$ and $\beta^{+}=\beta_{i}=\hat{\theta}\left(\phi_{*}, \eta_{*}\right)$.

Let $\epsilon>0$. It can be shown that $\hat{\theta}$ is continuous on $\mathcal{S} \times \mathbb{C}^{n \times l N}$. Thus, $\hat{\theta}\left(\phi_{*}, \cdot\right)$ is continuous on $\mathbb{C}^{n \times l N}$. Therefore, there exists $\delta \in\left(0, \delta_{0}\right)$ such that for all $\eta \in \mathbb{B}_{\delta}(0)$, $\hat{\theta}\left(\phi_{*}, \eta\right) \in \mathbb{B}_{\epsilon}\left(\hat{\theta}\left(\phi_{*}, 0\right)\right)$. Finally, assume $\left\|\eta_{*}\right\|<\delta$. Since $\beta^{+}=\hat{\theta}\left(\phi_{*}, \eta_{*}\right)$, it follows from $(20)$ that $\beta^{+} \in \mathbb{B}_{\epsilon}\left(\beta_{*}\right)$, which confirms (i).

To prove (ii), assume $\eta_{*}=0$. Thus, $\left\|\eta_{*}\right\|=0<\delta_{0}$ and part (i) implies that $\phi^{+}=\phi_{*}$. Since $\eta_{*}=0$, it follows from $(20)$ that $\beta^{+}=\hat{\theta}\left(\phi_{*}, 0\right)=\beta_{*}$.

Proof of Theorem 2. Let $\epsilon>0$. It can be shown that $\hat{\theta}$ is continuous on $\mathcal{S} \times \mathbb{C}^{n \times l N}$. Since, in addition, $\phi_{*} \in \mathcal{S}$, it follows that there exists $\delta_{0}>0$ such that for all $\phi \in \mathbb{B}_{\delta_{0}}\left(\phi_{*}\right)$ and all $\eta \in \mathbb{B}_{\delta_{0}}(0)$,

$$
\hat{\theta}(\phi, \eta) \in \mathbb{B}_{\epsilon}\left(\hat{\theta}\left(\phi_{*}, 0\right)\right) .
$$

Define $\epsilon_{1} \triangleq \min \left\{\epsilon, \delta_{0}\right\}$ and $\Lambda_{\mathrm{c}} \triangleq \overline{\Psi \cap \mathcal{S}_{\rho}}$. Using the process in [17, Prop. 8], it can be shown that $\Lambda_{\mathrm{c}} \subseteq \mathcal{S}$ is compact. Since $\Lambda_{\mathrm{c}}$ is compact, and $\left\{x \in \mathbb{R}^{a \times m}: \| x-\right.$ $\left.\phi_{*} \| \geq \epsilon_{1}\right\}$ is closed, it follows that $\Lambda_{\epsilon_{1}} \triangleq \Lambda_{\mathrm{c}} \backslash \mathbb{B}_{\epsilon_{1}}\left(\phi_{*}\right)=$ $\Lambda_{\mathrm{c}} \cap\left\{x \in \mathbb{R}^{a \times m}:\left\|x-\phi_{*}\right\| \geq \epsilon_{1}\right\}$ is compact. 
Let $c>\delta_{0}$, define $C \triangleq\left\{x \in \mathbb{C}^{n \times l N}:\|x\| \leq c\right\}$, and note that $\hat{Q}$ is continuous on $\Lambda_{\epsilon_{1}} \times C$. Next, define $\Theta: C \rightarrow[0, \infty)$ by $\Theta(\eta) \triangleq \min _{\phi \in \Lambda_{\epsilon_{1}}} \hat{Q}(\phi, \eta)$, which exists because $\Lambda_{\epsilon_{1}}$ is compact and $\hat{Q}$ is continuous on $\Lambda_{\epsilon_{1}} \times C$ [23, Thm. 7.7]. It follows from Proposition 3 and (A5) that $\Theta(0)>0$. Since $\hat{Q}$ is continuous on $\Lambda_{\epsilon_{1}} \times C$, and $\Lambda_{\epsilon_{1}}$ and $C$ are compact, it follows from [21, Thm. 9.14] that $\Theta$ is continuous on $C$. Furthermore, since $\hat{Q}$ is continuous on $\mathcal{S} \times \mathbb{C}^{n \times l N}$, it follows that $\hat{Q}\left(\phi_{*}, \cdot\right)$ is continuous on $C$. Thus, $W: C \rightarrow \mathbb{R}$ defined by $W(\eta) \triangleq$ $\Theta(\eta)-\hat{Q}\left(\phi_{*}, \eta\right)$ is continuous on $C$. Note that $(21) \mathrm{im}-$ plies that $W(0)=\Theta(0)-\hat{Q}\left(\phi_{*}, 0\right)=\Theta(0)>0$. Therefore, it follows that there exists $\delta_{1} \in(0, c)$ such that for all $\eta \in \mathbb{B}_{\delta_{1}}(0), W(\eta)>0$. Define $\delta \triangleq \min \left\{\delta_{0}, \delta_{1}\right\}>0$ and assume $\left\|\eta_{*}\right\|<\delta$. Then, $W\left(\eta_{*}\right)>0$.

Since $W\left(\eta_{*}\right)>0$ and $\hat{Q}\left(\cdot, \eta_{*}\right)$ is continuous on $\Lambda_{\mathrm{c}}$, it follows from the continuity of $\hat{Q}\left(\cdot, \eta_{*}\right)$ that there exists $\delta_{2}>0$ such that for all $\phi \in\left(\Lambda_{\mathrm{c}} \cap \mathbb{B}_{\delta_{2}}\left(\phi_{*}\right)\right), \mid \hat{Q}\left(\phi, \eta_{*}\right)-$ $\hat{Q}\left(\phi_{*}, \eta_{*}\right) \mid<W\left(\eta_{*}\right)$. Thus, for all $\phi \in\left(\Lambda_{\mathrm{c}} \cap \mathbb{B}_{\delta_{2}}\left(\phi_{*}\right)\right)$, $\hat{Q}\left(\phi, \eta_{*}\right)-\hat{Q}\left(\phi_{*}, \eta_{*}\right) \leq\left|\hat{Q}\left(\phi, \eta_{*}\right)-\hat{Q}\left(\phi_{*}, \eta_{*}\right)\right|<W\left(\eta_{*}\right)=$ $\Theta\left(\eta_{*}\right)-\hat{Q}\left(\phi_{*}, \eta_{*}\right)$, which implies that

$$
\hat{Q}\left(\phi, \eta_{*}\right)<\Theta\left(\eta_{*}\right) .
$$

Since $\left\{\Lambda_{j}\right\}_{j=1}^{\infty}$ converges to $\left(\Psi \cap \mathcal{S}_{\rho}\right) \subseteq \Lambda_{\mathrm{c}}$, it follows from Definition 1 that there exists a sequence $\left\{\phi_{j}: \phi_{j} \in\right.$ $\left.\Lambda_{j}\right\}_{j=1}^{\infty}$ and $L \in \mathbb{Z}^{+}$such that for all $j>L, \phi_{j} \in$ $\mathbb{B}_{\min \left\{\epsilon_{1}, \delta_{2}\right\}}\left(\phi_{*}\right)$. Thus, it follows from (23) that for all $j>L, \hat{Q}\left(\phi_{j}, \eta_{*}\right)<\Theta\left(\eta_{*}\right)$.

Let $j \in \mathbb{Z}^{+}$be such that $j>L$. It follows from Algorithm 1, (17)-(19), and (23) that $\hat{Q}\left(\phi_{j}^{+}, \eta_{*}\right) \leq$ $\hat{Q}\left(\phi_{j}, \eta_{*}\right)<\Theta\left(\eta_{*}\right)$. Assume for contradiction that $\phi_{j}^{+} \notin$ $\mathbb{B}_{\epsilon_{1}}\left(\phi_{*}\right)$, which implies that $\phi_{j}^{+} \in \Lambda_{\epsilon_{1}}$. Thus, $\Theta\left(\eta_{*}\right)=$ $\min _{\phi \in \Lambda_{\epsilon_{1}}} \hat{Q}\left(\phi, \eta_{*}\right) \leq \hat{Q}\left(\phi_{j}^{+}, \eta_{*}\right)<\Theta\left(\eta_{*}\right)$, which is a contradiction. Therefore, $\phi_{j}^{+} \in \mathbb{B}_{\epsilon_{1}}\left(\phi_{*}\right) \subseteq \mathbb{B}_{\epsilon}\left(\phi_{*}\right)$. Since $\phi_{j}^{+} \in \mathbb{B}_{\epsilon_{1}}\left(\phi_{*}\right) \subseteq \mathbb{B}_{\delta_{0}}\left(\phi_{*}\right)$, it follows from (20) and (22) that $\beta_{j}^{+}=\hat{\theta}\left(\phi_{j}^{+}, \eta_{*}\right) \in \mathbb{B}_{\epsilon}\left(\hat{\theta}\left(\phi_{*}, 0\right)\right)=\mathbb{B}_{\epsilon}\left(\beta_{*}\right)$.

\section{References}

[1] U. Forssell and L. Ljung. Closed-loop identification revisted. Automatica, 35:1215-1241, 1999.

[2] P. Van den Hof. Closed-loop issues in system identification. Annual Reviews in Control, 22:173-186, 1998.

[3] P. M. J. Van den Hof and R. J. P. Schrama. Identification and control-closed-loop issuses. Automatica, 31(12):1751-1770, 1995.

[4] R. Isermann and M. Münchhof. Identification of Dynamic Systems An Introduction with Applications. Springer, 2011.

[5] E. Roth, S. Sponberg, and N. J. Cowan. A comparative approach to closed-loop computation. Current opinion in neurobiology, 25:54-62, 2014.

[6] A. M. D'Amato, A. J. Ridley, and D. S. Bernstein. Retrospective-cost-based adaptive model refinement for the ionosphere and thermosphere. Statistical Analysis and Data Mining, 4:446-458, 2011.
[7] E. Itoh and S. Suzuki. Nonlinear approach for human internal models: Feedforward and feedback roles in pilot maneuver. In IEEE International Conference on Systems, Man and Cybernetics, volume 3, pages 2455-2462, 2005.

[8] F. M. Nieuwenhuizen, K. A. Beykirch, M. Mulder, and H. H. Bülthoff. Identification of pilot control behavior in a roll-lateral helicopter hover task. In AIAA Modeling and Simulation Technologies Conference and Exhibit, number AIAA-2007-6799, Hilton Head, SC, 2007.

[9] M. Olivari, F. M. Nieuwenhuizen, J. Venrooij, H. H. Bülthoff, and L. Pollini. Multi-loop pilot behavior identification in response to simultaneous visual and haptic stimuli. In $A I A A$ Modeling and Simulation Technologies Conference, number AIAA-2012-4795, Minneapolis, MN, 2012.

[10] F. M. Nieuwenhuizen and H. H. Bülthoff. The MPI CyberMotion simulator: A novel research platform to investigate human control behavior. Journal of Computing Science and Engineering, 7(2):122-131, 2013.

[11] C. C. Macadam. Understanding and modeling the human driver. Vehicle System Dynamics, 40(1-3):101-134, 2003.

[12] J. Steen, H. J. Damveld, R. Happee, M. M. van Paassen, and M. Mulder. A review of visual driver models for system identification purposes. In IEEE International Conference on Systems, Man, and Cybernetics, pages 20932100, Anchorage, AK, 2011.

[13] E. Hellstrom and M. Jankovic. A driver model for velocity tracking with look-ahead. In Proc. Amer. Contr. Conf., pages 3342-3347, Chicago, IL, 2015.

[14] T. Kiemel, Y. Zhang, and J. J. Jeka. Identification of neural feedback for upright stance in humans: Stabilizartion rather than sway minimization. Journal of Neuroscience, 31(42):15144-15153, 2011.

[15] F. M. Drop, D. M. Pool, H. J. Damveld, M. M. van Paassen, and M. Mulder. Identification of the feedforward component in manual control with predictable target signals. IEEE Trans. Cybernetics, 43(6):1936-1949, 2013.

[16] V. A. Laurense, D. M. Pool, H. J. Damveld, M. M. van Paassen, and M. Mulder. Effect of controlled element dynamics on human feedforward behavior in ramp-tracking tasks. IEEE Trans. Cybernetics, 45(2):253-265, 2015.

[17] X. Zhang and J. B. Hoagg. Frequency-domain subsystem identification with application to modeling human control behavior. Systems and Control Letters, 87:36-46, 2016.

[18] S. Gillijns and B. De Moor. Data-based subsystem identification for dynamic model updating. In Proc. Conf. Dec. Contr., pages 3303-3308, San Diego, CA, December 2006.

[19] H. J. Palanthandalam-Madapusi, S. Gillijns, B. De Moor, and D. S. Bernstein. Subsystem identification for nonlinear model updating. In Proc. Amer. Contr. Conf., pages 30563061, Minneapolis, MN, June 2006.

[20] A. V. Morozov, A. A. Ali, A. M. D'Amato, A. J. Ridley, S. L. Kukreja, and D. S. Bernstein. Retrospective-costbased model refinement for system emulation and subsystem identification. In Proc. Conf. Dec. Contr., pages 2142-2147, Orlando, FL, December 2011.

[21] R. K. Sundaram. A First Course in Optimization Theory. Cambridge University Press, Cambridge, 1996.

[22] W. Ford. Numerical Linear Algebra with Applications: Using MATLAB. Academic Press, 2014.

[23] R. Beals. Analysis An Introduction. Cambridge University Press, Cambridge, 2004. 